02

\title{
Поляризационные измерения комбинационного рассеяния света в слоях кремния на сапфире
}

\author{
(C) A.B. Иго \\ Ульяновский государственный университет, \\ 432063 Ульяновск, Россия \\ e-mail: igoalexander@mail.ru
}

Поступила в редакцию 09.11.2017 г.

В окончательной редакции 15.03.2018 г.

\begin{abstract}
Проведены измерения интенсивности поляризованных компонент комбинационного рассеяния света для различных кристаллографических направлений в эпитаксиальных слоях кремния на сапфире. Эксперимент показывает, что рассеянное излучение имеет значительную деполяризованную составляющую, которая напрямую связана с дефектностью эпитаксиального слоя. Для описания дефектного кристаллического слоя кремния на сапфире предложена модель ансамбля кристаллитов. Показано, что по отношению интенсивностей поляризованной и деполяризованной компонент можно определить характерную величину разупорядочения кристаллических доменов в эпитаксиальном слое. По измерениям интенсивности комбинационного рассеяния света в зависимости от кристаллографического направления определена анизотропия тензора комбинационного рассеяния света. Получены численные значения для двух образцов.
\end{abstract}

DOI: $10.21883 /$ OS.2018.07.46262.261-17

\section{Введение}

Эпитаксиальные слои кремния на сапфире (КНС) имеют важное применение в микроэлектронике для производства радиационно-стойких приборов [1] и микроэлектронных датчиков давления [2]. Спектры комбинационного рассеяния света (КРС) в эпитаксиальном слое кремния показывают отличие от спектров монокристаллического (объемного) кремния. Отличия проявляются в сдвиге максимума спектральной линии и увеличении ее ширины. Эти особенности объясняются повышенной дефектностью и наличием упругих деформаций в эпитаксиальном слое кремния в области границы с сапфировой подложкой. По спектрам КРС были определены величины этих деформаций [3], а параметры дефектности кристаллической решетки хоть и прямо влияют на ширину спектральной линии КРС, но традиционно измеряются методом дифракции рентгеновских лучей (XRD) $[1,4]$.

Технологические исследования в области создания КНС-структур с низкодефектными эпитаксиальными слоями кремния и толщинами порядка $100 \mathrm{~nm}$ и менее продолжаются $[1,4]$. Дефектность и деформированное состояние кремниевого слоя прямо влияют на его электрофизические свойства и должны учитываться при проектировании приборов [2,5]. Основными методами оценки качества кремниевого слоя являются методы XRD и просвечивающей электронной микроскопии (ПЭМ) [1].

КРС в кристалле монокристаллического кремния поляризовано и описывается тензором КРС, который имеет вид [6]:

$$
\begin{gathered}
R(x)=\left(\begin{array}{lll}
0 & 0 & 0 \\
0 & 0 & d \\
0 & d & 0
\end{array}\right), \quad R(y)=\left(\begin{array}{lll}
0 & 0 & d \\
0 & 0 & 0 \\
d & 0 & 0
\end{array}\right), \\
R(z)=\left(\begin{array}{lll}
0 & d & 0 \\
d & 0 & 0 \\
0 & 0 & 0
\end{array}\right) .
\end{gathered}
$$

Символами $x, y, z$ обозначены поляризации трехкратно вырожденного оптического фонона в центре зоны Бриллюэна кремния. Такой вид имеет тензор КРС в гармоническом приближении, но в реальном дефектном кристалле гармоническое приближение невзаимодействующих колебательных мод уже недостаточно. В случае дефектного кристалла некоторые компоненты тензора могут стать ненулевыми, а в эксперименте будет присутствовать доля деполяризованного КРС за счет уменьшения интенсивности поляризованного КРС. В работе [7] приведена методика определения компонент тензора КРС для произвольно ориентированных кристаллов по данным поляризационных измерений.

В настоящей работе ставится задача экспериментально и теоретически изучить влияние дефектности кристаллической решетки на значения компонент тензора КРС на примере структур КНС.

\section{Тензор КРС ансамбля кристаллитов}

Интенсивность рассеянного света, поляризованного по направлению $\mathbf{e}^{s}$, определяется тензором (1) и поля- 


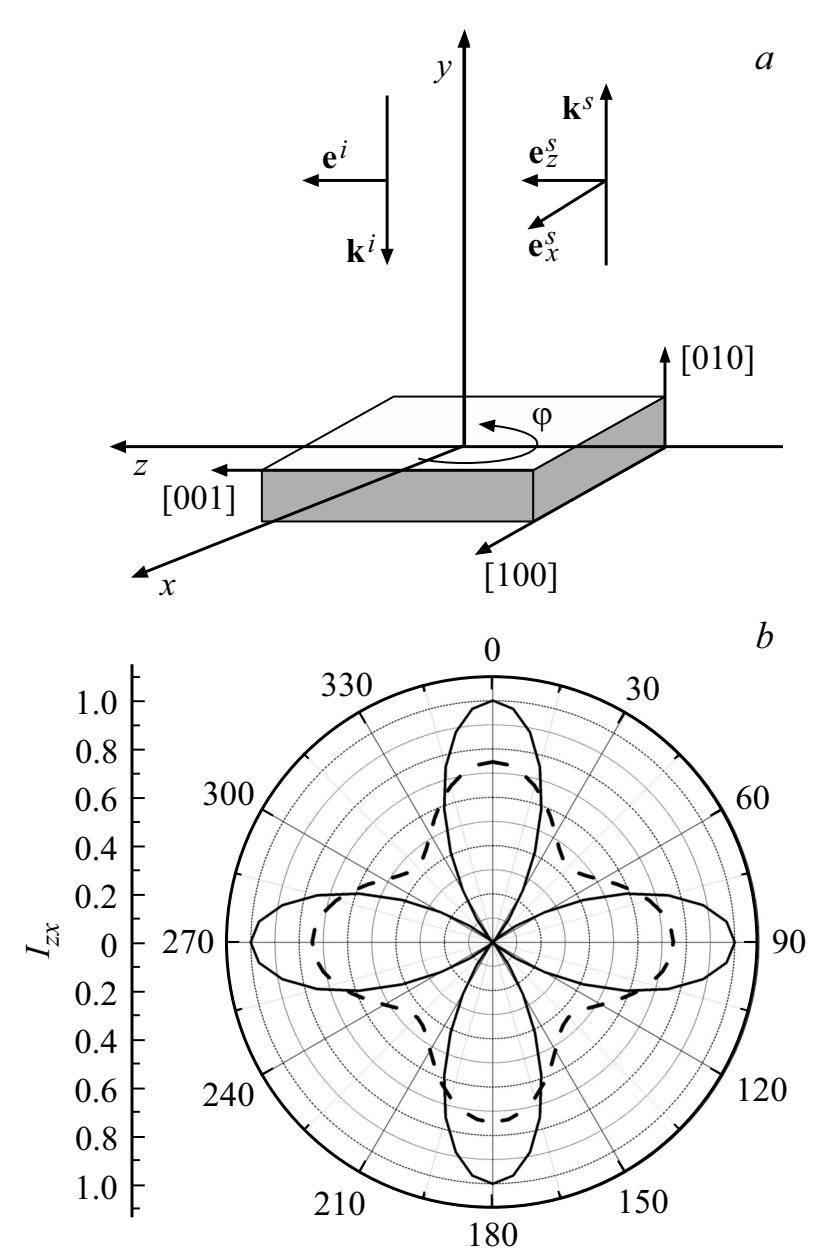

Рис. 1. Рассеяние света в слое кремния в лабораторной системе координат. $a-$ положение образца и направления падающего и рассеянного света. $b$ - зависимость интенсивности компоненты $I_{z x}$ рассеянного света от угла поворота образца $\varphi$. Сплошная линия - для монокристаллического образца. Штриховая линия - для ансамбля кристаллитов с дисперсией $\sigma^{2}=0.17$.

ризацией падающего света $\mathbf{e}^{i}$ по формуле [6]

$$
I=A \sum_{\alpha}\left[\mathbf{e}^{i} \mathbf{R}(\alpha) \mathbf{e}^{s}\right]^{2}
$$

Рассмотрим рассеяние света в лабораторной системе координат $x, y, z$ в геометрии обратного рассеяния, как показано на рис. 1. Зададим направление падающего света по оси $y$, поляризацию падающего света по оси $z$ : $\mathbf{e}^{i}=\left(\begin{array}{ll}0 & 01\end{array}\right)$, а рассеянный свет будем регистрировать в направлении оси $y$ поочередно в двух плоскостях поляризации, по оси $z: \mathbf{e}_{z}^{s}=\left(\begin{array}{lll}0 & 0 & 1\end{array}\right)$ и по оси $x: \mathbf{e}_{x}^{s}=\left(\begin{array}{lll}1 & 0 & 0\end{array}\right)$. Зарегистрированные интенсивности рассеянного света в этом случае обозначим как $I_{z z}$ и $I_{z x}$. Расположим кристалл так, что его главные оси совпадают с лабораторной системой координат, а затем повернем его на угол $\varphi$, как показано на рис. 1.

Если $U_{\varphi}^{y}-$ матрица поворота вокруг оси $y$ на угол $\varphi$, тогда тензор (1) в лабораторной системе коор- динат становится равным $R_{\varphi}(\alpha)=U_{\varphi}^{y} R(\alpha) \tilde{U}_{\varphi}^{y}$. Проводя вычисления (2) для выбранных условий эксперимента, получим угловую зависимость компонент интенсивности для двух поляризаций (константа А принята равной 1):

$$
\begin{aligned}
I_{z x} & =\sum_{\alpha}\left[\mathbf{e}^{i} R_{\varphi}(\alpha) \mathbf{e}_{z}^{s}\right]^{2}=d^{2} \cos ^{2} 2 \varphi, \\
I_{z z} & =\sum_{\alpha}\left[\mathbf{e}^{i} R_{\varphi}(\alpha) \mathbf{e}_{x}^{s}\right]^{2}=d^{2} \sin ^{2} 2 \varphi .
\end{aligned}
$$

Пусть в области светового пятна падающего света присутствуют кристаллиты (кристаллические области) с объемом $V_{i}$ и углом отклонения от среднего направления $\theta_{i}$. В этом случае зарегистрированная интенсивность поляризованных компонент будет равна сумме интенсивностей от отдельных областей:

$$
\begin{gathered}
I_{z x}=d^{2} \sum_{i} V_{i} \cos ^{2} 2\left(\varphi-\theta_{i}\right), \\
I_{z z}=d^{2} \sum_{i} V_{i} \sin ^{2} 2\left(\varphi-\theta_{i}\right) .
\end{gathered}
$$

Предложим, что кристаллиты примерно одного размера имеют некоторое разупорядочение с дисперсией $\sigma$ относительно среднего направления, совпадающего с кристаллографическим направлением, т.е. описываются нормальным распределением $P(\sigma, 0, \theta)$. Заменяя суммирование интегрированием, в приближении $\sigma<1 \mathrm{c}$ помощью программы Wolfram Mathematica получим

$$
\begin{aligned}
I_{z x} & =\frac{d^{2} V}{\sqrt{2 \pi} \sigma} \int_{-\pi}^{\pi} \exp \left(-\theta^{2} / 2 \sigma^{2}\right) \cos ^{2} 2(\varphi-\theta) d \theta \\
& \cong d^{2} V \frac{1}{2}\left(1+\exp \left(-8 \sigma^{2}\right) \cos (4 \varphi)\right)
\end{aligned}
$$

Обозначим $a=\exp \left(-8 \sigma^{2}\right)$, и приводя к единичному объему $(V=1)$ преобразуем (5) к виду

$$
\begin{aligned}
I_{z x} & =d^{2} \frac{1}{2}(1+a \cos (4 \varphi)) \\
& =d^{2} \frac{1+a}{2} \cos ^{2} 2 \varphi+d^{2} \frac{1-a}{2} \sin ^{2} 2 \varphi .
\end{aligned}
$$

Аналогично получим значение для второй компоненты в (4)

$$
\begin{aligned}
I_{z z} & =d^{2} \frac{1}{2}(1-a \cos (4 \varphi)) \\
& =d^{2} \frac{1+a}{2} \sin ^{2} 2 \varphi+d^{2} \frac{1-a}{2} \cos ^{2} 2 \varphi .
\end{aligned}
$$

При $a=1$ рассеяние полностью поляризовано и описывается тензором (1) монокристалла. При $a=0$ рассеяние полностью деполяризовано и соответствует рассеянию света в поликристаллах со случайно ориентированными кристаллитами в плоскости $(x, z)$. Угловая 
зависимость интенсивностей (6) и (7) для случая произвольного значения степени поляризации соответствует следующему виду тензора КРС:

$$
\begin{gathered}
R(x)=\left(\begin{array}{ccc}
b & 0 & 0 \\
0 & 0 & d \\
0 & d & -b
\end{array}\right), \quad R(y)=\left(\begin{array}{ccc}
0 & 0 & e \\
0 & 0 & 0 \\
e & 0 & 0
\end{array}\right), \\
R(z)=\left(\begin{array}{ccc}
b & d & 0 \\
d & 0 & 0 \\
0 & 0 & -b
\end{array}\right) .
\end{gathered}
$$

Этот вид тензора соответствует ситуации, когда часть колебательной энергии из моды, поляризованной по направлению $y$, перетекает в колебательные моды с поляризациями $x$ и $z$. Новые компоненты $e$ и $b$ равны

$$
e=d \frac{\sqrt{1+a}}{\sqrt{2}}, \quad b=d \frac{\sqrt{1-a}}{\sqrt{2}} .
$$

На рис. 1 приведены графики расчета угловой зависимости компоненты интенсивности КРС (6) для $a=0$ (совершенный монокристалл) и $a=0.25$, что соответствует среднеквадратичному разбросу направлений кристаллитов $\sigma^{2}=0.17$.

\section{Тензор КРС при учете рассеяния фононов на дефектах кристаллической решетки}

Согласно рис. 1, в геометрии обратного рассеяния волновые векторы падающего и рассеянного света коллинеарные. Вид тензора КРС (1) и уравнение (2) приводят к результату, что рассеяние света наблюдается только на оптических фононах, имеющих поляризацию, направленную вдоль направления распространения (ось $y$ ), т.е. на продольных оптических фононах. Такие фононы имеют в лабораторной системе координат волновой вектор $\mathbf{q}=(0 q 0)$ и направление вектора поляризации $\mathbf{e}_{q}=\left(\begin{array}{lll}0 & 1 & 0\end{array}\right)$.

Невзаимодействующие фононы в гармоническом приближении описываются плоскими волнами, распространяющимися в направлении оси $y$, но в случае рассеяния на удалении от рассеивающего центра, волна приобретает сферическую составляющую [8]

$$
\Psi_{q}=e^{i \mathbf{q y}}+\frac{f(\theta)}{r} e^{i \mathbf{q r}} .
$$

Амплитуда рассеяния $f(\theta)=f$ для медленных частиц не зависит от угла рассеяния [8]. Нормируем (10) из условия $C^{2} \int\left|\Psi_{q}(r)\right|^{2} d V=1$, тогда интенсивность рассеянного света (2) состоит из двух составляющих

$$
I=C^{2}\left[\sum_{\alpha}\left[\mathbf{e}^{i} \mathbf{R}_{\varphi}(\alpha) \mathbf{e}^{s}\right]^{2}+4 \pi f^{2} \sum_{\alpha}\left[\mathbf{e}^{i} \mathbf{R}(\alpha) \mathbf{e}^{s}\right]^{2}\right] .
$$

В силу сферической симметрии рассеянной волны интенсивность рассеянного света второй составляющей не зависит от угла поворота кристалла, т.е. является постоянной величиной в лабораторной системе координат и может быть рассчитана для тензора КРС в главных осях (1). Задавая поляризацию падающего $\mathbf{e}^{i}=\left(\begin{array}{lll}0 & 0 & 1\end{array}\right)$ и рассеянного света $\mathbf{e}_{z}^{s}=\left(\begin{array}{lll}0 & 0 & 1\end{array}\right)$, из (11) получим

$$
\begin{aligned}
I_{z x} & =C^{2}\left(d^{2} \cos ^{2} 2 \varphi+4 \pi f^{2} d^{2}\right) \\
& =C^{2} d^{2}\left(1+4 \pi f^{2}\right) \cos ^{2} 2 \varphi+C^{2} d^{2} 4 \pi f^{2} \sin ^{2} 2 \varphi .
\end{aligned}
$$

Аналогично для второй компоненты

$$
\begin{aligned}
I_{z z} & =C^{2}\left(d^{2} \sin ^{2} 2 \varphi+4 \pi f^{2} d^{2}\right) \\
& =C^{2} d^{2}\left(1+4 \pi f^{2}\right) \sin ^{2} 2 \varphi+C^{2} d^{2} 4 \pi f^{2} \cos ^{2} 2 \varphi .
\end{aligned}
$$

При $f=0$ рассеяние полностью поляризовано и описывается тензором (1) монокристалла. При $f>0$ рассеяние частично деполяризовано. Для произвольного значения $f$ тензор КРС в главных осях имеет вид (8), где компоненты $e$ и $b$ равны

$$
e=d \frac{\sqrt{1+4 \pi f^{2}}}{c}, \quad b=d \frac{\sqrt{4 \pi} f}{c} .
$$

Измерение степени деполяризации в эксперименте позволит определить параметр дефектности кристалла $f$ или $\sigma$ в зависимости от того, какой моделью дефектный кристалл описывается. Величине $\sigma^{2}=0.17$ соответствует $f=0.34$.

\section{Эксперимент и сравнение с расчетами}

Измерение КРС проводили для двух образцов КНС, представляющих собой пластины диаметром $76 \mathrm{~mm} \mathrm{c}$ толщинами слоя кремния 0.92 и $0.64 \mu \mathrm{m}$, и для референтного образца монокристалла кремния на модернизированном спектрометре ДФС-52 с ФЭУ Hamamatsu Н6240-01, для возбуждения КРС использовался лазер с длиной волны $532 \mathrm{~nm}$. Схема эксперимента приведена на рис. 2. В эксперименте измерялись интенсивности КРС двух поляризаций рассеянного света для последовательно задаваемых значений угла поворота образца $\varphi$ с шагом $10^{\circ}$.

Согласно рис. 2, излучение от лазера направлялось вдоль оси $z$ и имело поляризацию $\mathbf{e}^{i}$ по оси $y$. Угол между поверхностью образца и направлением падения луча в эксперименте оставался постоянным и был равен $\psi=0.275$. Преломленный луч, согласно закону преломления, распространяется в кремнии под углом $\psi^{\prime}$ относительно нормали к образцу, и вектор поляризации в лабораторной системе отсчета приобретает компоненты $\mathbf{e}^{i}=\left(0, \sin \left(\psi+\psi^{\prime}\right), \cos \left(\psi+\psi^{\prime}\right)\right)$. Рассеянное излучение измерялось в направлении $y$ с поляризацией вдоль направлений $z: \mathbf{e}_{z}^{s}=\left(\begin{array}{lll}0 & 0 & 1\end{array}\right), \mathbf{e}_{x}^{s}=\left(\begin{array}{lll}1 & 0 & 0\end{array}\right)$. Измеренные интенсивности обозначены как $I_{y z}$ и $I_{y x}$. Они приведены в виде экспериментальных точек на рис. 3 для монокристаллического кремния и для структуры КНС толщиной слоя кремния $0.92 \mu \mathrm{m}$. 


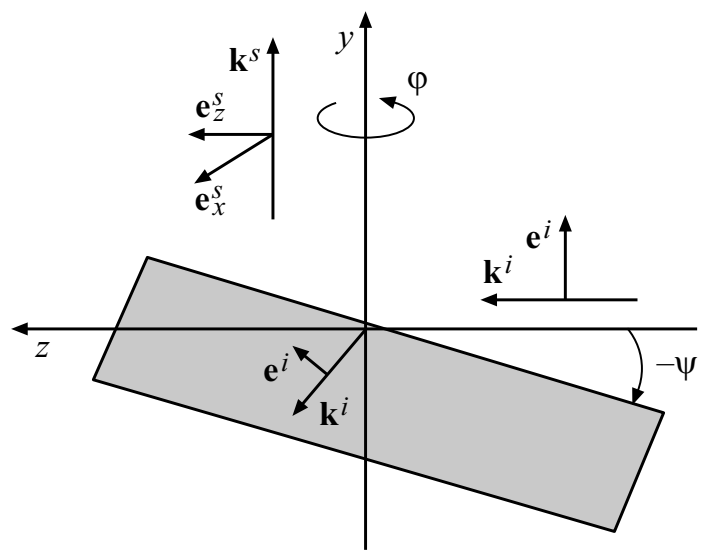

Рис. 2. Схема измерения рассеяния света в КНС структуре. Показано положение образца в плоскости $(y, z)$, направление оси $x$ перпендикулярно плоскости рисунка. Угол $\varphi$ отсчитывается от положительного направления оси $x$. Стрелками показаны направления падающего и рассеянного света и их поляризации.

Проводя последовательно повороты вокруг оси $y$ на угол $\varphi$ и вокруг оси $x$ на угол $-\psi$, получим, что тензор (1) в лабораторной системе координат становится равным

$$
R_{\varphi}(\alpha)=U_{-\psi}^{x} U_{\varphi}^{y} R(\alpha) \tilde{U}_{\varphi}^{y} \tilde{U}_{-\psi}^{x} .
$$

Проводя вычисления (2) с тензором (15) с помощью программы Wolfram Mathematica, получим для выбранных условий эксперимента угловую зависимость компонент интенсивности для двух поляризаций:

$$
\begin{aligned}
I_{y x}(\varphi) & =\sum_{\alpha}\left[\mathbf{e}^{i} R_{\varphi}(\alpha) \mathbf{e}_{z}^{s}\right]^{2}=d^{2} \cos ^{2} \psi^{\prime} \cos ^{2} 2 \varphi+d^{2} \sin ^{2} \psi^{\prime}, \\
I_{y z}(\varphi) & =\sum_{\alpha}\left[\mathbf{e}^{i} R_{\varphi}(\alpha) \mathbf{e}_{x}^{s}\right]^{2} \\
& =d^{2} \cos ^{2} \psi^{\prime} \cos ^{2} \psi \sin ^{2} 2 \varphi+d^{2} \sin ^{2}\left(\psi-\psi^{\prime}\right) .
\end{aligned}
$$

Воспользуемся этими формулами для описания экспериментальных результатов, приведенных на рис. 3 . Используя (5), получим скорректированные значения интенсивностей (16):

$$
\begin{gathered}
I_{y x}^{\prime}(\varphi)=\int_{-\pi}^{\pi} P(\sigma, 0, \theta) I_{y x}(\varphi-\theta) d \theta, \\
I_{y z}^{\prime}(\varphi)=\int_{-\pi}^{\pi} P(\sigma, 0, \theta) I_{y z}(\varphi-\theta) d \theta, \\
I_{y x}^{\prime}(\varphi)=d^{2} \cos ^{2} \psi^{\prime} \frac{1}{2}(1+a \cos (4 \varphi))+d^{2} \sin ^{2} \psi^{\prime}, \\
I_{y z}^{\prime}(\varphi)=d^{2} \cos ^{2} \psi^{\prime} \cos ^{2} \psi \cos ^{2} \psi \\
\times \frac{1}{2}(1-a \cos (4 \varphi))+d^{2} \sin ^{2}\left(\psi-\psi^{\prime}\right) .
\end{gathered}
$$

Наилучшим образом эксперимент для монокристалла описывается (18) с параметром $a=0.91$, что соответствует $\sigma^{2}=0.01$. Расчетная кривая с этим значением приведена на рис. 3 в виде сплошной линии. Это значение параметра соответствует суммарной ошибке, вносимой в эксперимент за счет эллиптичности поляризации лазерного луча, неточности выставления углов поляризации и неидеальности поляризатора.

Эксперимент для КНС описывается $I_{y x}^{\prime}(\varphi)$ с параметром $a=0.29$, что соответствует $\sigma^{2}=0.15$, а для $I_{y z}^{\prime}(\varphi)$ - с параметром $a=0.39$, что соответствует $\sigma^{2}=0.12$. Расчетные кривые с этими значениями приведены на рис. 3 в виде сплошных линий. Разные значения $\sigma^{2}$ для двух направлений отражают наличие анизотропии в структуре КНС.

Величина асимметрии значительно усиливается в пластине КНС со слоем кремния $0.64 \mu \mathrm{m}$. Измерения компонент поляризации приведены на рис. 4. Наличие асимметрии в угловой зависимости интенсивности КРС
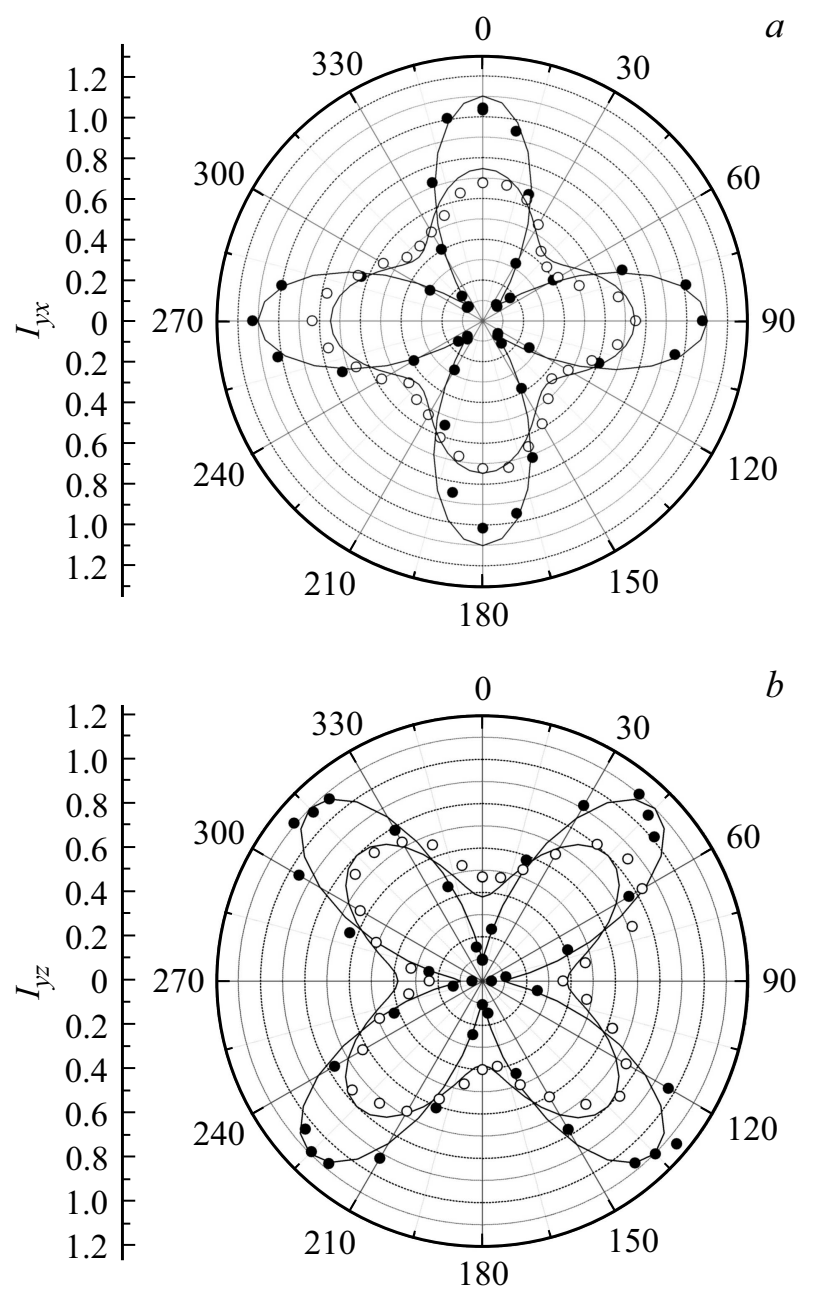

Рис. 3. Зависимость интенсивности поляризованных компонент рассеянного света от угла поворота образца $\varphi$. Сплошная линия - расчет, точки - эксперимент: - - монокристалл кремния, о- структура КНС с толщиной слоя кремния $0.92 \mu$ m. $a-I_{y x}(\varphi), b-I_{y z}(\varphi)$. 


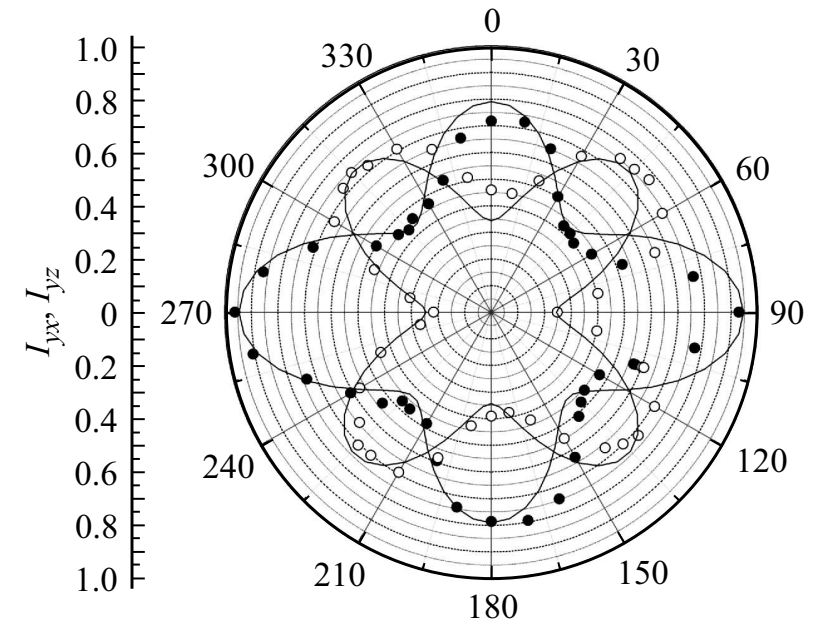

Рис. 4. Зависимость интенсивности поляризованных компонент рассеянного света в зависимости от угла поворота образца $\varphi$ в структуре КНС с толщиной слоя кремния $0.64 \mu \mathrm{m}$. Сплошные линии - расчет, точки - эксперимент: черные точки - компонента $I_{y x}(\varphi)$, светлые кружки - компонента $I_{y z}(\varphi)$.

возможно, когда компоненты тензора (1) для разных поляризаций отличаются. Рассогласование кристаллических решеток кремния и сапфира в направлении [001] $\mathrm{Si} \|$ [1101] $\mathrm{Al}_{2} \mathrm{O}_{3}$ составляет $4.4 \%$, а в направлении [100] $\mathrm{Si} \|[\overline{1} \overline{1} 20] \mathrm{Al}_{2} \mathrm{O}_{3}$ 12.3\% [4]. Деформация кристаллического слоя кремния приводит к понижению симметрии его кристаллической решетки. В эксперименте по оси $y$ лабораторной системы координат (совпадает при $\psi=0$ c $[010] \mathrm{Si}$ ) упругая деформация отсутствует, а по осям $z$ и $x$ (в плоскости пластины) - присутствует и разная. Симметрия кубической кристаллической решетки кремния $O_{h}$ в этом случае понизится до $D_{2 h}$ [9], а тензор КРС будет иметь вид [6]

$$
\begin{gathered}
R(x)=\left(\begin{array}{ccc}
0 & 0 & 0 \\
0 & 0 & d_{x} \\
0 & d_{x} & 0
\end{array}\right), \quad R(y)=\left(\begin{array}{ccc}
0 & 0 & d_{y} \\
0 & 0 & 0 \\
d_{y} & 0 & 0
\end{array}\right) \\
R(z)=\left(\begin{array}{ccc}
0 & d_{z} & 0 \\
d_{z} & 0 & 0 \\
0 & 0 & 0
\end{array}\right) .
\end{gathered}
$$

Проводя вычисления (2) с тензором (19), получим для выбранных условий эксперимента угловую зависимость компонент интенсивности для двух поляризаций:

$$
\begin{aligned}
I_{y x}(\varphi)=d_{y}^{2} & \cos ^{2} \psi^{\prime} \cos ^{2} 2 \varphi+\left(d_{z}^{2}+\left(d_{x}^{2}-d_{z}^{2}\right) \sin ^{2} \varphi\right) \sin ^{2} \psi^{\prime} \\
I_{y z}(\varphi)= & d_{y}^{2} \cos ^{2} \psi^{\prime} \cos ^{2} \psi \sin ^{2} 2 \varphi \\
& +\left(d_{z}^{2}+\left(d_{x}^{2}-d_{z}^{2}\right) \cos ^{2} \varphi\right) \sin ^{2}\left(\psi-\psi^{\prime}\right)
\end{aligned}
$$

Подставляя уравнения (20) в модель ансамбля кристаллитов (17), получим скорректированные значения интенсивностей:

$$
\begin{aligned}
& I_{y x}^{\prime}(\varphi)=d_{y}^{2} \cos ^{2} \psi^{\prime} \frac{1}{2}\left(1+\exp \left(-8 \sigma^{2}\right) \cos (4 \varphi)\right) \\
& +\left[d_{z}^{2}+\left(d_{x}^{2}-d_{z}^{2}\right) \frac{1}{2}\left(1+\exp \left(-2 \sigma^{2}\right) \cos (2 \varphi)\right)\right] \sin ^{2} \psi^{\prime} \\
& I_{y z}^{\prime}(\varphi)=d_{y}^{2} \cos ^{2} \psi^{\prime} \cos ^{2} \psi \frac{1}{2}\left(1-\exp \left(-8 \sigma^{2}\right) \cos (4 \varphi)\right) \\
& +\left[\left(d_{z}^{2}+\left(d_{x}^{2}-d_{z}^{2}\right) \frac{1}{2}\left(1-\exp \left(-2 \sigma^{2}\right) \cos (2 \varphi)\right)\right] \sin ^{2}\left(\psi-\psi^{\prime}\right) .\right.
\end{aligned}
$$

Сделаем упрощающее предположение, что значение $d_{z}$ (по направлению наименьшей деформации) не сильно отличается от недеформированного значения $d_{y}$, тогда нормированные зависимости (21) имеют 2 подгоночных параметра: $\sigma^{2}$ и $k=\frac{d_{x}^{2}-d_{z}^{2}}{d_{y}^{2}}$. Результат подгонки при $k=3$ и $\sigma^{2}=0.1$ для $I_{y x}^{\prime}(\varphi)$ и $\sigma^{2}=0.09$ для $I_{y z}^{\prime}(\varphi)$ представлен на рис. 4 сплошными линиями. Наилучшая подгонка для пластины КНС толщиной $0.92 \mu \mathrm{m}$ дает значения $k=2$ и $\sigma^{2}=0.15$ для $I_{y x}^{\prime}(\varphi)$ и $\sigma^{2}=0.1$ для $I_{y z}^{\prime}(\varphi)$. Логично, что для более толстого слоя кремния величина $k$ уменьшается. Величина $\sigma^{2}$, как и в более простой модели (18), остается зависимой от кристаллографического направления.

Величина $\sigma^{2}$ имеет интегральный характер и показывает относительную долю кристаллитов в кремниевом слое, имеющих разброс ориентации. Качественно такая модель согласуется с данными, полученными методом дифракции рентгеновских лучей в структурах КНС $[1,4]$. В работе [1] в слое кремния в направлении [100] были выявлены домены с ориентацией [110] и домены [111] c ориентацией $14^{\circ}$ к подложке. С помощью ПЭМ выявлены двойники с границами двойникования параллельно и перпендикулярно границе раздела. Двойники наблюдались по всей толщине кремниевого слоя. Более того, каких-либо областей без двойников не было выявлено. По данным [4] эпитаксиальный слой кремния на сапфировой подложке начинает расти в виде отдельных островков, которые, увеличиваясь в объеме, создают сплошной слой кремния.

Кривые дифракционного отражения (КДО) кремния характеризуются значительной шириной, определенной в эксперименте [1] для разных (отдельных) рефлексов в диапазоне 2025-2950 угловых секунд, т.е. порядка $0.5-1^{\circ}$. В то же время общую (интегральную) величину разброса для выделенного направления метод не дает. Для целей оценки электрофизических свойств полупроводника важно знать именно интегральные значения дефектности слоя. Метод КРС и модель разупорядоченных кристаллитов, представленная в настоящей работе, дают суммарную величину разброса порядка $10^{\circ}$, зависящую от направления и исследуемой пластины. Можно сказать, что данные сопоставимы, учитывая то, что образцы КНС, исследованные в экспериментах, были существенно разные. 
На рис. 4 видно, что экспериментальные точки, соответствующие максимумам при $0^{\circ}, 45^{\circ}, 125^{\circ}, 180^{\circ}$, имеют смещение порядка $5^{\circ}$ относительно расчетных значений. Такое смещение согласуется с данными ПЭМ [1] о том, что направление [110] кремния смещено на $4^{\circ}$ относительно кристаллографического направления подложки [22̄01]. В эксперименте структура КНС ориентировалась в лабораторной системе координат по базовому срезу подложки.

\section{Выводы}

В работе экспериментально исследованы угловые зависимости интенсивности поляризованных компонент КРС в структурах кремний на сапфире. Для описания экспериментальных данных предложена модель ансамбля кристаллитов. Модель предполагает нормальное распределение разброса направлений одинаковых кристаллитов. По данным измерений была рассчитана дисперсия разброса для двух пластин КНС разной толщины.

Эксперимент показывает, что тензор КРС в деформированном слое кремния приобретает более сложную структуру по сравнению с монокристаллом. Показано, что компоненты тензора КРС связаны с величиной и направлением деформации в структуре КНС. Получена аналитическая формула интенсивности КРС с учетом коэффициента анизотропии и величины разупорядочения кристаллической решетки. Результаты эксперимента и расчета сопоставлены с данными по дифракции рентгеновских лучей в структурах КНС.

Полученные результаты можно использовать для оценки дефектности структур кремний на сапфире.

\section{Список литературы}

[1] Благов А.Е., Васильев А.Л., Голубева А.С., Иванов И.А., Кондратьев О.А., Писаревский Ю.В., Пресняков М.Ю., Просеков П.А., Серегин А.Ю. // Кристаллография. 2014. T. 59. № 3. C. 356.

[2] Стучебников В.М. // Радиотехника и электроника. 2005. T. 50. № 6. C. 678 .

[3] Anastassakis E., Pinczuk A., Burstein E., Pollak F.H., Cardona M. // Sol. Stat. Commun. 1970. V. 8. P. 133.

[4] Павлов Д.А., Шиляев П.А., Коротков Е.В., Кривулин Н.О. // Письма в ЖТФ. 2010. Т. 36. № 12. С. 16.

[5] De Wolf I. // Semicond. Sci. Technol. 1996. V. 11. P. 139.

[6] Loudon R. // Adv. Phys. 1964. V. 13. P. 423.

[7] Munisso M.C., Zhy W., Pezzotti G. // Phys. Stat. Sol. B. 2009. V. 246. N 8. P. 1893.

[8] Ландау Л.Д., Лифшии, Е.М. Квантовая механика. М.: Наука, 1974. 752 с.

[9] Бир Г.Л., Пикус Г.Е. Симметрия и деформационные эффекты в полупроводниках. М.: Наука, 1972. 584 с. 